\title{
Use of KikGR a photoconvertible green-to-red fluorescent protein for cell labeling and lineage analysis in ES cells and mouse embryos
}

\author{
Sonja Nowotschin and Anna-Katerina Hadjantonakis*
}

Address: Developmental Biology Program, Sloan-Kettering Institute, New York, NY 10065, USA

Email: Sonja Nowotschin - nowotscs@mskcc.org; Anna-Katerina Hadjantonakis* - hadj@mskcc.org

* Corresponding author

Published: 9 September 2009

BMC Developmental Biology 2009, 9:49 doi:10.1186/147/-213X-9-49

This article is available from: http://www.biomedcentral.com/I47I-213X/9/49

(C) 2009 Nowotschin and Hadjantonakis; licensee BioMed Central Ltd.

This is an Open Access article distributed under the terms of the Creative Commons Attribution License (http://creativecommons.org/licenses/by/2.0), which permits unrestricted use, distribution, and reproduction in any medium, provided the original work is properly cited.

\begin{abstract}
Background: The use of genetically-encoded fluorescent proteins has revolutionized the fields of cell and developmental biology and in doing so redefined our understanding of the dynamic morphogenetic processes that shape the embryo. With the advent of more accessible and sophisticated imaging technologies as well as an abundance of fluorescent proteins with different spectral characteristics, the dynamic processes taking place in situ in living cells and tissues can now be probed. Photomodulatable fluorescent proteins are one of the emerging classes of geneticallyencoded fluorescent proteins.
\end{abstract}

Results: We have compared PA-GFP, PS-CFP2, Kaede and KikGR four readily available and commonly used photomodulatable fluorescent proteins for use in ES cells and mice. Our results suggest that the green-to-red photoconvertible fluorescent protein, Kikume Green-Red (KikGR), is most suitable for cell labeling and lineage studies in ES cells and mice because it is developmentally neutral, bright and undergoes rapid and complete photoconversion. We have generated transgenic ES cell lines and strains of mice exhibiting robust widespread expression of KikGR. By efficient photoconversion of KikGR we labeled subpopulations of ES cells in culture, and groups of cells within ex utero cultured mouse embryos. Red fluorescent photoconverted cells and their progeny could be followed for extended periods of time.

Conclusion: Transgenic ES cells and mice exhibiting widespread readily detectable expression of KikGR are indistinguishable from their wild type counterparts and are amenable to efficient photoconversion. They represent novel tools for non-invasive selective labeling specific cell populations and live imaging cell dynamics and cell fate. Genetically-encoded photomodulatable proteins such as KikGR represent emergent attractive alternatives to commonly used vital dyes, tissue grafts and genetic methods for investigating dynamic behaviors of individual cells, collective cell dynamics and fate mapping applications. 


\section{Background}

Cell fate, pattern formation and morphogenesis depend on dynamic cell interactions involving a multitude of cell behaviors and cell populations. One way to gain insight into these events is to label and observe single or groups of cells over time. Several approaches have been established for labeling cells in developing mouse embryos, the mammalian genetically-tractable model of choice, including dye injections, electroporation of nucleic acids or proteins into single or groups of cells $[1,2]$ as well as grafting of genetically-distinct tissues $[3,4]$ or using chimeras [57]. Unfortunately, most of these techniques are invasive, and only effective when the tissue or cells of interest are easy accessible for manipulation. Therefore, it has been a challenge to tag single or groups of cells that are not superficially located.

The advent of genetically-encoded fluorescent proteins has afforded the ability to label cells ubiquitously or selectively depending on the cis-regulatory elements used in transgene design. Whereas native fluorescent proteins are cytosolic [8], subcellularly-localized fluorescent proteins can be used for higher resolution image information [9]. For example, fluorescent proteins fused to a human histone $\mathrm{H} 2 \mathrm{~B}$, since they are bound to active chromatin, allow the visualization and tracking of individual cells within a group [10-12]. Likewise, fluorescent protein fusions that localize to the plasma membrane provide information on membrane dynamics and cell morphology $[11,13]$. Thus in combination with increasingly sophisticated imaging technology, for example laser scanning confocal or multiphoton microscopy, and on-stage cultures, geneticallyencoded fluorescent proteins can provide high resolution information on dynamic cell behaviors.

Binary genetic approaches such as genetically inducible fate mapping (GIFM) based on the Cre/loxP system can also be used for cell labeling and fate mapping in mice. GIFM is non-invasive and allows the tagging and tracking of non-superficial cell types [14]. However, cell type specificity relies on availability of cis-regulatory elements to drive Cre recombinase transgene expression within specific populations of cells. To date, binary genetic methods have been used to gain information on large groups of cells rather than smaller groups or even individual cells in spatially-defined regions of interest (ROIs). Therefore, methods that combine lineage labeling through transgenesis with spatially-defined ROIs are likely to provide greater flexibility in cell labeling and lineage tracing.

Photomodulatable fluorescent proteins should help overcome the limitations of these approaches by enabling non-invasive selective labeling of cells in ROIs. Various photomodulatable fluorescent proteins including PA-GFP which acquires fluorescence upon activation, PS-CFP2 which changes color from cyan to green fluorescence upon activation, as well as Kaede, KikGR and EosFP which go from a green to a red fluorescence upon activation, have been used to study cell behaviors in different organisms, including Drosophila, chick, Xenopus and zebrafish [15-19], with preliminary proof-of-principle applications recently reported in mice $[20,21]$. For example, an alphatubulin PA-GFP fusion has used to investigate the dynamics of mesoderm cell migration during Drosophila development [18], and KikGR has been used to study the migratory behaviors of neural crest cells in chick embryos [22].

With so many available photomodulatable proteins, choice of the most suitable for any given application and in any particular organism is not obvious as few direct comparisons have been reported. An extensive comparison of PA-GFP, PS-CFP2, Kaede and KikGR for their applicability in cell migratory behavior and cell lineage analysis in the chick embryo has been reported [23]. Importantly, applications in mice are in their infancy and to date are limited one study in pre-implantation embryos [21] and the characterization of adult transgenic mice. Transgenic strains expressing Kaede have been described in two independent studies $[20,24]$. One of these studies investigated the movement of cells of the immune system and employed flow cytometry to analyze photoconverted cells [20]. Therefore, live cell imaging experiments using photomodulatable reporters have yet to be fully explored in mice.

Recognizing the need for a comparison of commonly used photomodulatable fluorescent proteins for their prospective use in mice, we compared PA-GFP, PS-CFP2, Kaede and KikGR. We found the green-to-red photoconvertible protein KikGR, engineered from the coral Favia favus [25], to be the best suited for cell labeling and fate mapping due to its specific and efficient photoconversion and outstanding brightness compared to the other photomodulatable fluorescent proteins tested in this study. We therefore generated and characterized transgenic mouse strains constitutively expressing KikGR under the CAG promoter [26]. We also report the generation of transgenic ES cell lines exhibiting widespread expression of KikGR. We observed bright fluorescence in KikGR transgenic ES cells, embryos and adult mice. We noted rapid and efficient photoconversion of KikGR at various developmental stages. To study cell dynamics in mouse embryos, groups of cells in spatially defined ROIs were photoconverted and followed over time.

This study therefore provides the first comparison of photomodulatable fluorescent proteins for use in ES cells and mice, and reveals the potential utility of these geneticallyencoded reporters for investigating individual cell behaviors, population dynamics and cell fate in the mouse embryo. 


\section{Results and Discussion Photoconversion of cells and ex utero culture of embryos to visualize cell dynamics in situ}

Since their spectral properties can be modulated in defined regions of interest, photoconvertible fluorescent proteins provide greater control of labeling specificity

A PHOtoconVERSION IN CELLS

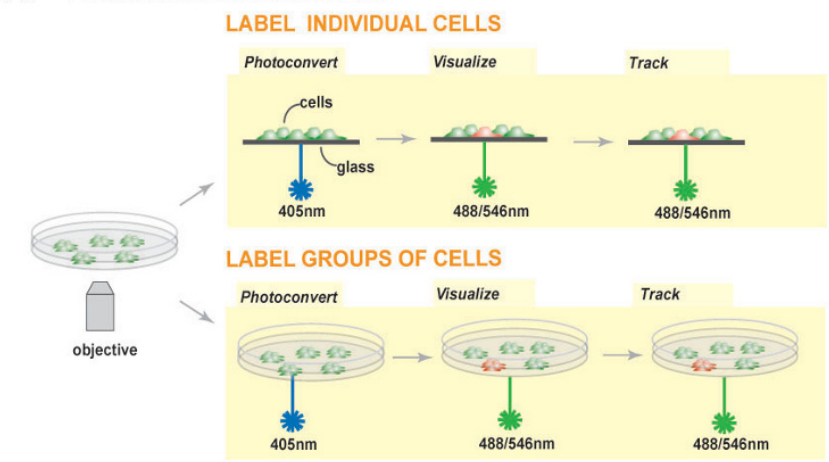

B PHOTOCONVERSION IN THE EMBRYO

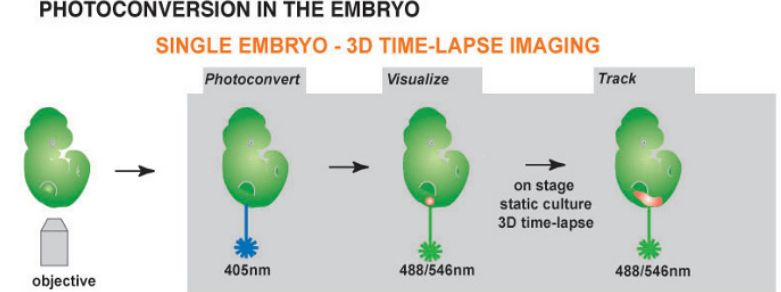

MULTIPLE EMBRYOS - 3D IMAGING AT VARIOUS TIME-POINTS

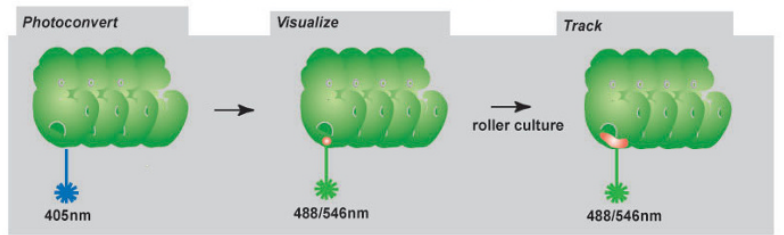

Figure I

Schematic representation of the concept of photoconversion of cells in culture and in embryos. Photoconversion in cells (A): Single cells or group of cells expressing a constitutively active KikGR can be labeled noninvasively. KikGR expressing cells fluoresce green before photoconversion. After exposure to short wavelength light $(405 \mathrm{~nm})$ a single or a group of cells of interest can be photoconverted. Photoconverted cells emit red fluorescence and can be tracked over time. Photoconversion in mouse embryos (B): Any KikGR-positive cell or group of cells of interest in a mouse embryo can be photoconverted using short wavelength light ( $405 \mathrm{~nm}$ ). Photoconverted red fluorescent cells can be imaged in live static cultured embryos, so that 3D over time (4D) data sets can be acquired. Using live imaging and roller culture, a region of interest (ROI) in multiple embryos (for example all embryos in one litter) can be photoconverted in the same experiment and imaged. This is advantageous for comparing cell behavior in wild type and mutant mice before any visible onset of a phenotype. over conventional fluorescent proteins. They are therefore superior for precisely and non-invasively labeling single or small cohorts of cells in complex populations (Figure $1 \mathrm{~A}$ ). Our goal was to generate transgenic ES cells and mice with widespread expression of a readily detectable and efficiently photoconvertible fluorescent protein reporter. These would be used for 3D time-lapse imaging cell dynamics in situ in ES cell cultures and mouse embryos. Once determined wild type cell behaviors can be contrasted with those resulting from experimental perturbations, for example in mutants. By using photoconversion in combination with ex utero embryo culture cells can be tracked over time providing a way to visualize the cell dynamics that direct the emergence of morphologically distinct structures or mutant phenotypes (Figure 1B).

\section{Construction and evaluation of different photomodulatable proteins in cells}

To establish if one of a series of the widely available photomodulatable proteins would be better suited for lineage studies in mouse embryos, we compared PA-GFP [27], PSCFP2 [28], Kaede [29] and KikGR [25]. All four photomodulatable proteins were cloned under the widespread CAGGS enhancer/promoter, comprising the chicken betaactin promoter and first exon/intron placed downstream of the CMV immediate early enhancer [26]. COS-7 and ES cells were transfected with the four CAG promoter-based plasmids and visualized pre- and post photoactivation. Results are summarized in Table 1. Comparison of expression and photoactivation or photoconversion, respectively, in cells, showed high sensitivity of PA-GFP to UV light and rapid photoactivation. However, even exposure to daylight resulted in instantaneous autoactivation or bleaching of the PA-GFP fluorophore. This was unfavourable since mouse embryos develop in utero and must be dissected from maternal tissues using a stereo dissecting microscope. They therefore require illumination of both the microscope stage and animal for removal of the uterus. PS-CFP2 was dim, and its photoconversion was inefficient. Furthermore, given the spectral overlap between the cyan and green forms of the protein, to optimally distinguish photoconverted and unconverted cells linear unmixing of spectra is necessary (Figure 2). Kaede was photoconverted efficiently but was dim compared to KikGR (Figure 2). Kaede also often formed aggregates in COS-7 cells and was toxic in ES cells when expressed at high levels (data not shown). By contrast, photoconversion of KikGR was immediate and very efficient. Furthermore, KikGR did not appear to be toxic when highly expressed in ES cells, and was the brightest of the four evaluated photomodulatable proteins. These features made KikGR the protein of choice for use in ES cells and, by extrapolation, in mouse embryos.

We next established transgenic ES cell lines constitutively expressing KikGR. Fluorescent transgenic ES cell colonies 
Table I: Comparison of genetically-encoded photomodulatable fluorescent proteins.

\begin{tabular}{|c|c|c|c|}
\hline Fluorescent reporter & Color pre-activation & Color post-activation & Comments \\
\hline PA-GFP & Non-fluorescent & green & Considerable autoactivation (i.e. photoactivation through daylight). \\
\hline PS-CFP2 & cyan & green & $\begin{array}{l}\text { Dim pre-activation. Inefficient photoconversion. Considerable overlap } \\
\text { between pre- and post-activation spectral profiles. }\end{array}$ \\
\hline Kaede & green & red & $\begin{array}{l}\text { Dim. Efficient photoconversion. Aggregates form in COS cells, toxic at } \\
\text { when expressed at high levels in ES cells. }\end{array}$ \\
\hline KikGR & green & red & Bright pre- and post-activation. Efficient photoconversion. \\
\hline
\end{tabular}

were identified by their green fluorescence and picked under an epifluorescence stereo dissecting microscope. Clones were expanded and passaged in 96-well plates, and scored for the maintenance and level of green fluorescent reporter after extended maintenance in culture in the absence of selection. Clones that failed to meet these criteria were discarded from further analysis. The photocon- version and photoefficiency of KikGR was quantified in CAG::KikGR transgenic ES cells.

\section{Photoconversion of KikGR in ES cells}

Imaging of photoconversion in an ES cell colony constitutively expressing KikGR using short wavelength laser light $(405 \mathrm{~nm})$ revealed complete and fast photoconversion of

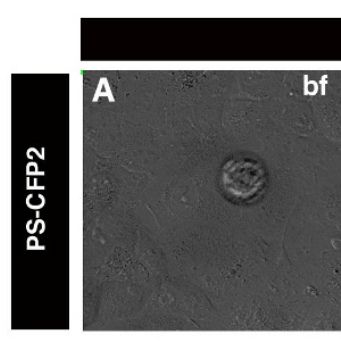

\section{pre-photoconversion}

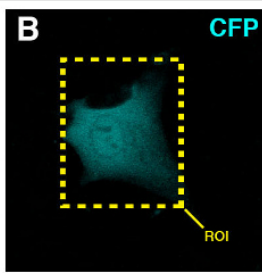

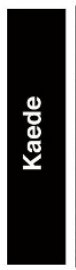
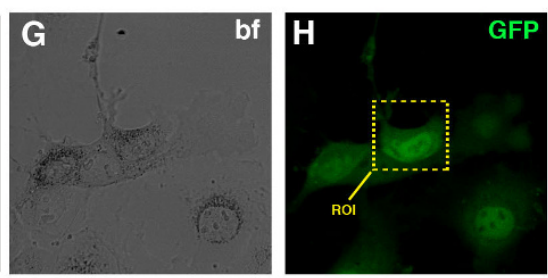

兘
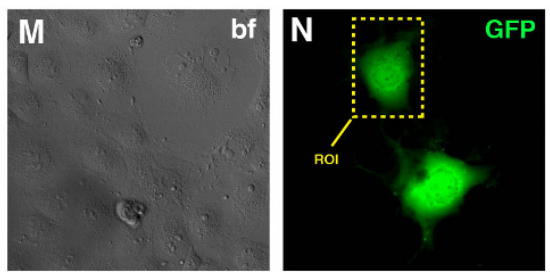
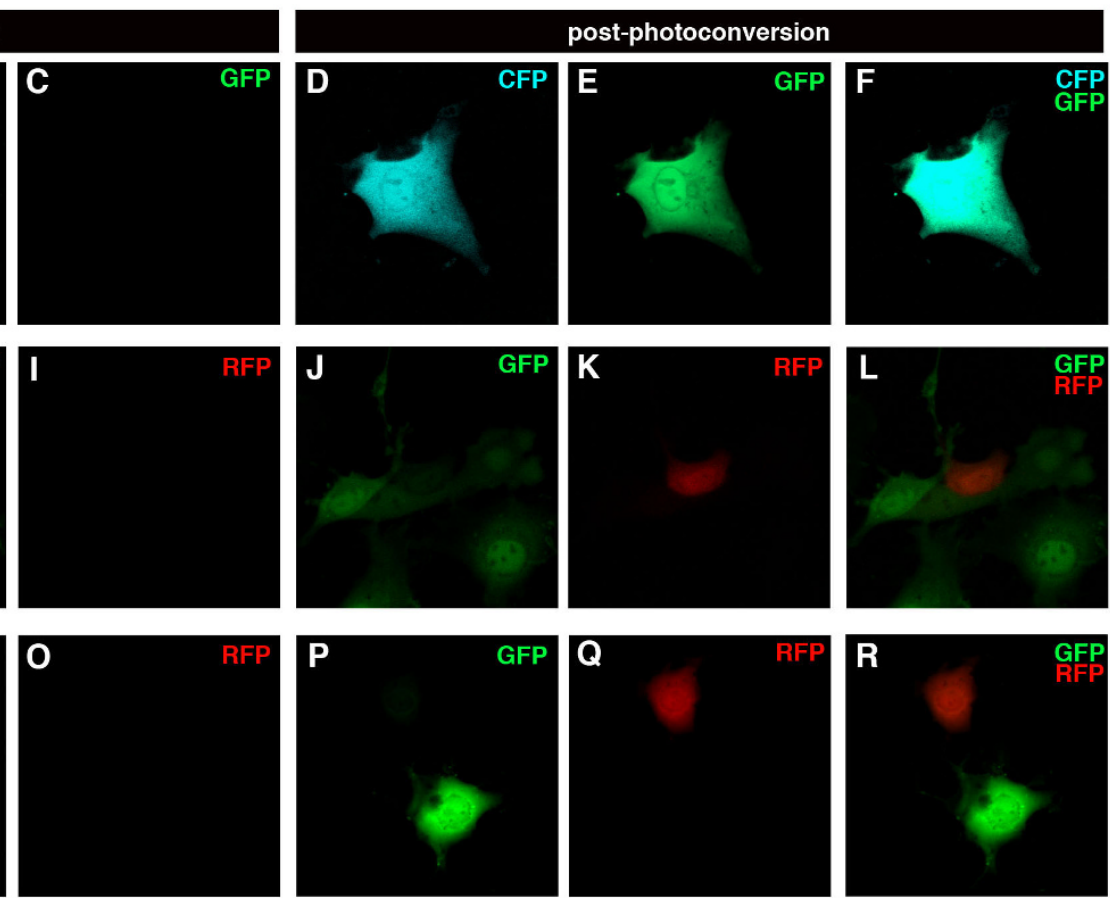

Figure 2

Comparative photoconversion of PS-CFP2, Kaede and KikGR. Panels show photoconversion of PS-CFP2 (A-F), Kaede $(G-H)$ and KikGR (M-R) in transient transfected COS7 cells. The ROI that was subjected to short wavelength laser light is depicted in the yellow dashed box. Bright field (BF) images of respective cell transfections (A, $G$ and $M$ ). Bright field images are $2 \mathrm{D}$ representations (A, G and M). Panels B-F, H-L and N-R show 3D images of converted COS7 cells. Upper row shows photoconversion of PS-CFP2. Cells fluoresce cyan before photoconversion and green after photoconversion. CFP channel (B) and GFP channel (C) before photoconversion. CFP (D) and GFP channel (E) after photoconversion. Merge of the CFP and GFP channel (F). Middle row depicts the photoconversion of Kaede, and bottom row shows the photoconversion of KikGR both of which fluoresce green before, and red after photoconversion. GFP channel $(\mathrm{H}, \mathrm{N})$ and RFP channel $(\mathrm{l}, \mathrm{O})$ before photoconversion. GFP (J, P) and RFP channel (K, Q) after photoconversion. Merge of the GFP and RFP channels (L, R). Note that you see a stronger CFP signal in D due to signal bleed through of the GFP channel. Note that cells were photoconverted with a 20x objective using 35\% laser power of the $25 \mathrm{~mW} 405 \mathrm{~nm}$ laser, however, KikGR was imaged with less gain in the channels of its respective colors as compared to PS-CFP2 and Kaede. 


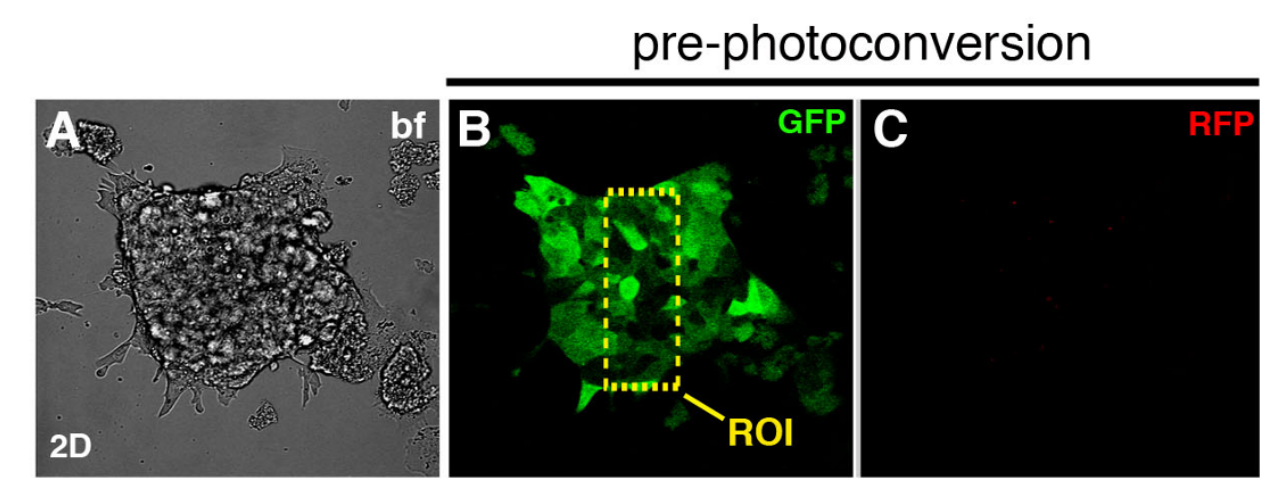

post-photoconversion
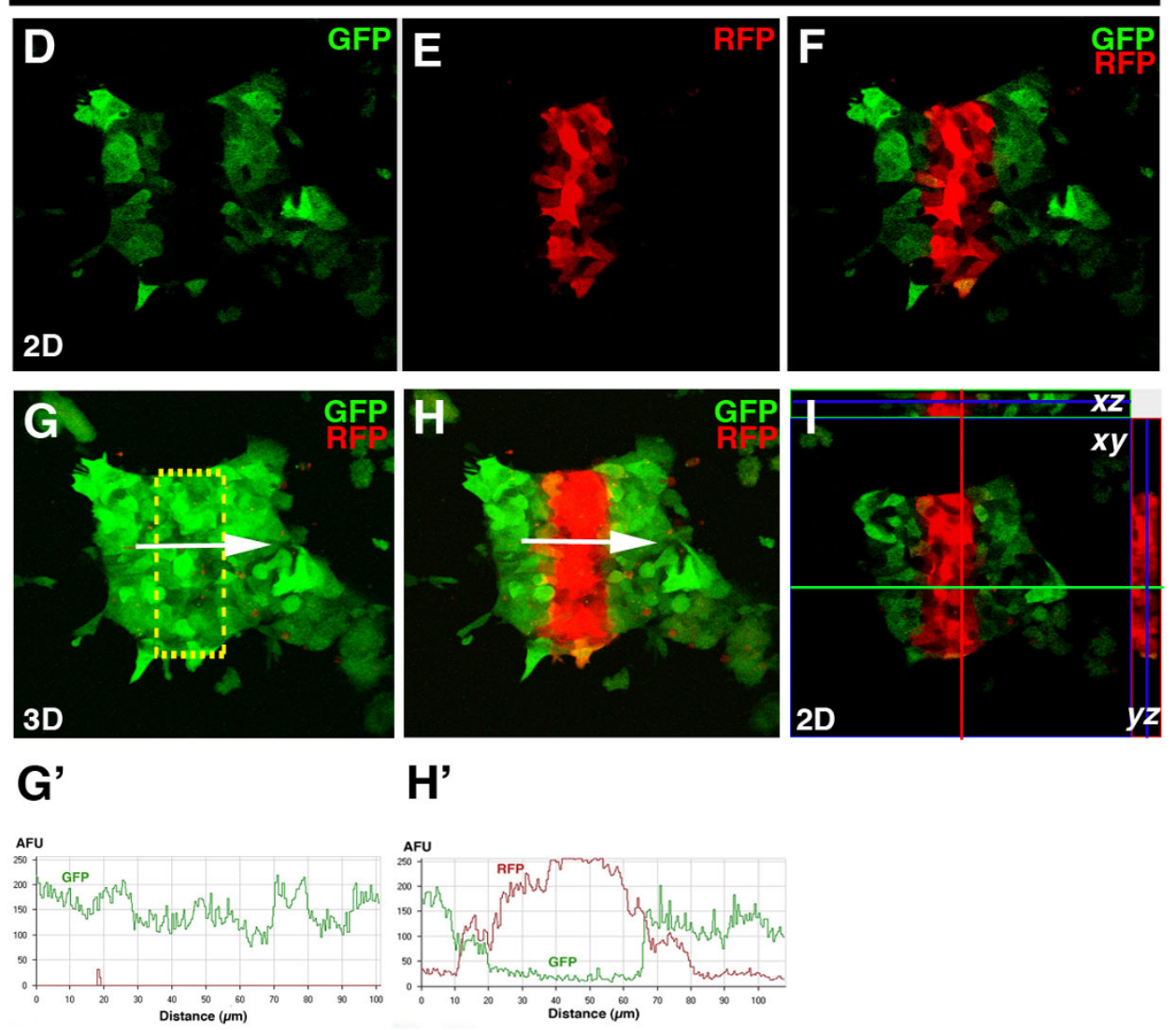

Figure 3

Photoconversion of widespread expressing CAG::KikGR transgenic ES cells. Panels show 2D (A-F, I) and 3D images $(G, H)$ of the photoconversion of a selected group of cells in an ES cell colony. Bright field image of ES cell colony (A). Image of ES cell colony with green $(B)$ and red $(C)$ channel showing green but no red fluorescence prior to photoconversion. Yellow dashed box show regions of interest (ROI) that is to be photoconverted. After photoconversion, cells in an ROI do not fluoresce green $(D)$ but red $(E)$. Merge of green and red channel after photoconversion, showing complete photoconversion of cells in the region of interest. 3D image of ES cell colony before photoconversion. All cells emit green fluorescence (G). Line plot showing average fluorescent intensity measured along the white arrow in G (G'). 3D image of ES cell colony after photoconversion. Photoconverted cells emit red fluorescence $(\mathrm{H})$. Line plot showing average fluorescent intensity measured along the white arrow in $\mathrm{H}$ showing complete photoconversion of cells, i.e. shift of emission spectrum from green to red in photoconverted region $\left(\mathrm{H}^{\prime}\right)$. 2D orthogonal representation of photoconverted ES cell colony showing complete photoconversion in the region of interest through entire z-stack (I). 
KikGR from green to red fluorescence (Figures 3 and 4) Any ROI could be defined and efficiently photoconverted without compromising cell viability. Complete photoconversion was demonstrated by imaging $z$-stacks of photoconverted ES cells (Figure 3A-F). 2D orthogonal representation of $z$-stack of ES cell colony shows that only photoconverted cells with no residual green fluorescence reside within the square $\mathrm{ROI}$ in sections taken throughout the $z$-stack (Figure 3I). Line plots illustrating the average fluorescent intensity along a line plotted across an ROI before and after photoconversion demonstrated a complete shift of emission from green to red fluorescence (Figure $\left.3 \mathrm{G}, \mathrm{G}^{\prime} \mathrm{H}, \mathrm{H}^{\prime}\right)$.

Exposure of a circular ROI defined within in an ES cell colony to $405 \mathrm{~nm}$ light (30\% laser power, $25.0 \mathrm{~mW}$ ) demonstrated fast photoconversion of KikGR (Figure 4A-E). Complete photoconversion was reached in 79 seconds under the experimental conditions used. Continued exposure of photoconverted cells eventually lead to photobleaching of the fluorophore and a slight reduction in red fluorescence (Figure 4G-H). Fluorescent intensities of the green and red channel plotted over time illustrated the rapid shift in the emission spectrum from $516 \mathrm{~nm}$ to 593 $\mathrm{nm}$ after exposure to $405 \mathrm{~nm}$ light (Figure 4I).

\section{Expression and function of KikGR in adult transgenic mice and post-implantation embryos}

The CAG::KikGR construct was used to generate transgenic strains of mice with widespread expression of KikGR. Three founders were characterized. Each line exhibited widespread expression, however, all data presented in this article derive from a single line. To characterize the $C A G:: K i k G R$ mouse strains and confirm that they exhibited widespread transgene expression as detected by green fluorescence, adult organs and post-implantation embryos of various stages were imaged using wide field epifluorescent microscopy (Figures 5 and 6). Dissection of organs from a 6 week old adult $C A G:: K i k G R^{T g} /+$ animal, and its wild type littermate revealed robust widespread expression of KikGR in organs of the hemizygous transgenic (Figure 5A-F). In addition, embryos of post-implantation stages ranging from embryonic (E) day 7.5 through to E12.5 exhibited strong widespread green fluorescence (Figure 6). Photoconversion of an ROI in the brain of an $C A G:: K i k G R^{T g /+}$ using a UV filter and epifluorescent illumination demonstrated the functionality of the KikGR protein in the transgenic mouse (Figure 5G-L). Importantly, CAG::KikGR ${ }^{T g /+}$ animals were indistinguishable from their littermates, both as embryos and adults, suggesting that readily detectable levels of KikGR expression are developmentally neutral and non-toxic.

\section{Live imaging population dynamics using KikGR}

Coordinated behaviors within cell populations are integral to a wide variety of morphogenetic events. Using transgenesis to direct KikGR expression in embryos and photoconversion of ROIs in combination with 3D timelapse imaging and ex utero embryo culture, the collective cell behaviors directing the morphogenesis of the mouse embryo can be probed. For example, given the precision of cell labeling using defined ROIs, it can be determined if collective cell behaviors such as convergence and extension occur in mouse embryos. A convergence and exten-
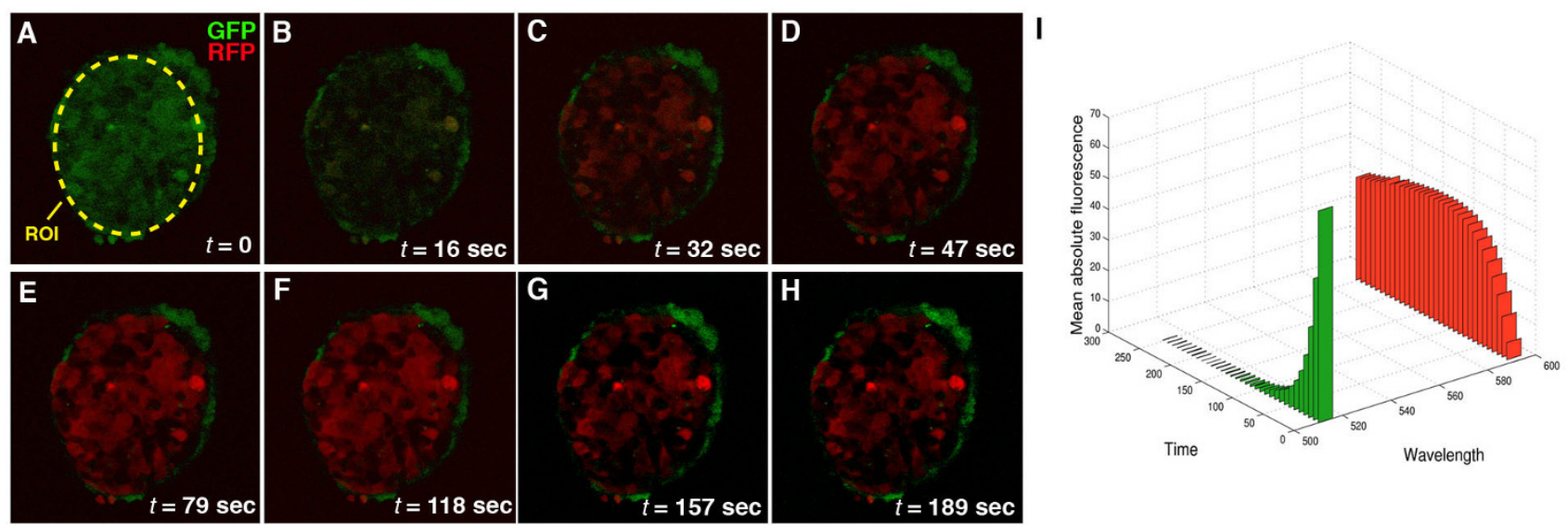

Figure 4

Efficiency of KikGR photoconversion over time with constant exposure to short wavelength laser light. Continuous scanning over time with $405 \mathrm{~nm}$ laser light excitation ( $30 \%$ laser power; $25.0 \mathrm{~mW}$ ) of an ES cell colony, constitutively expressing KikGR, shows fast and complete photoconversion. Fluorescence in green and red channels at different times of 405 $\mathrm{nm}$ exposure $(\mathrm{A}-\mathrm{H})$. Yellow dashed ellipse depicts region of interest (ROI) exposed to $405 \mathrm{~nm}$ laser light. Plot of time series in A-H shows shift in emission spectra from $513 \mathrm{~nm}$ to $596 \mathrm{~nm}(\mathrm{I})$. 


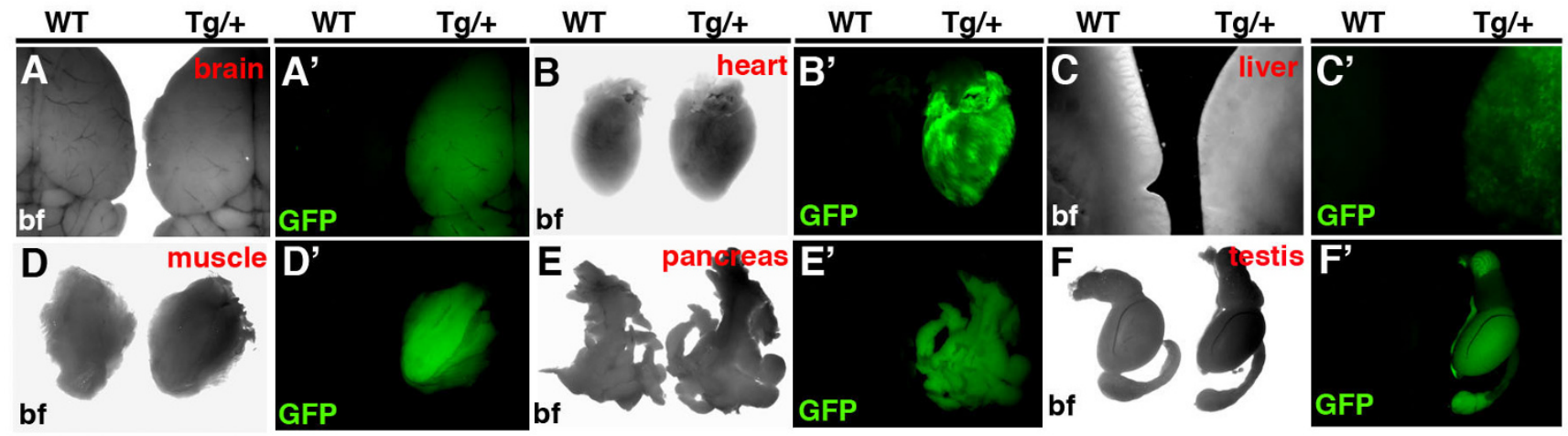

pre-photoconversion

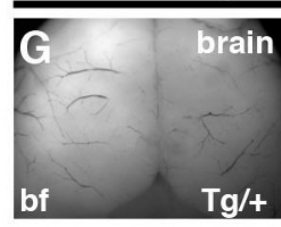

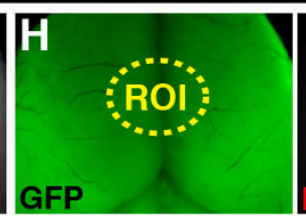

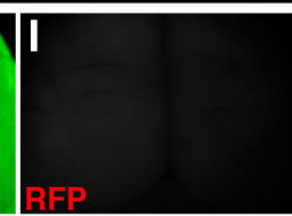

post-photoconversion

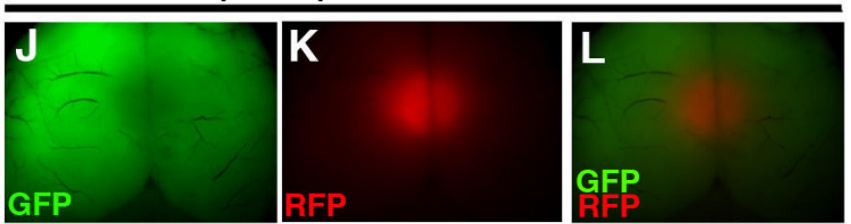

Figure 5

Widespread KikGR expression in organs of CAG::KikGRTg/+ adult mice. Panels of bright field and corresponding dark field epifluorescent images of organs taken from a 6 week old CAG::KikGRTg/+ mouse and a non-transgenic littermate. Brain (A, $\left.A^{\prime}\right)$, heart $\left(B, B^{\prime}\right)$, liver $\left(C, C^{\prime}\right)$, muscle $\left(D, D^{\prime}\right)$, pancreas $\left(E, E^{\prime}\right)$ and testis $\left(F, F^{\prime}\right)$. Panels of bright field and corresponding dark field epifluorescent images (green and red channels) of the brain before and after photoconversion using a UV filter (G-L). Yellow dashed ellipse depicts region of interest $(\mathrm{ROI})$ to be photoconverted $(\mathrm{H})$. Green channel $(\mathrm{H}, \mathrm{J})$. Red channel $(\mathrm{I}, \mathrm{K})$. Overlay of images of green and red channel (L).

sion type cell behavior can be investigated through photoconversion of cells contained in a rectangular ROI, and measurement of the height-to-width over time. One can then determine if the starting ratio is maintained or skews in any particular dimension over time, with the latter trend suggestive of a convergent extension type cell behavior.

Figure 7 shows a posterior-ventral view of an E8.5 ( 6 somite stage) embryo. By photoconversion of an ROI of known dimensions within the caudal part of the unsegmented (presomitic) paraxial mesoderm (Figure 7A-D), and subsequent imaging after a 6-hour period of roller culture, the dimensions (height-to-width ratio) of the photoconverted cell population were seen to change over time (Figure 7E-H). This change in the height-to-width ratio ( $N=3$ embryos) suggests that cells of the unsegmented paraxial mesoderm of a 6 -somite stage mouse embryo may undergo a convergent extension type collective cell behavior, an observation that warrants further investigation.

\section{Live imaging of cell fate in the mouse embryo}

Next, to determine if $C A G:: K i k G R$ transgenic mice were suitable for following cell fates, we investigated the first steps during cardiac morphogenesis (Figure 8). A small group of $(<30)$ cells residing on the right side of the car- diac crescent of an E8.0 embryo were photoconverted (Figure 8A-E). After $12 \mathrm{hrs}$ in roller culture, during which time cells of the cardiac crescent fuse at the midline and go on to form a linear heart tube, the photoconverted cells and/or their progeny became incorporated in, and restricted to, the right side of the linear heart tube (Figure $8 \mathrm{~F}-\mathrm{G}$ ). These results reveal that cells located on the right side of the cardiac crescent only contribute to the right side of the linear heart tube [30]. These data demonstrate that photomodulatable fluorescent protein reporters should facilitate precise labeling and fate mapping of defined cell populations during mouse embryonic development.

\section{Tracking cells over time using 3D time-lapse imaging and on-stage mouse embryo culture}

Ultimately, time-lapse image acquisition provides the highest resolution information on cell dynamics. Therefore, we next assessed if we could photoconvert a group of cells and track them in situ in embryos that were cultured on a microscope stage as opposed to in a roller culture incubator [31]. Our data suggest that, as with roller culture, on-stage static embryo culture as well as multiple exposures to laser light at timed intervals did not adversely affect embryonic development. Still frames of the bright field channel of a 3D time-lapse imaging experiment show development of a mouse embryo cultured static 


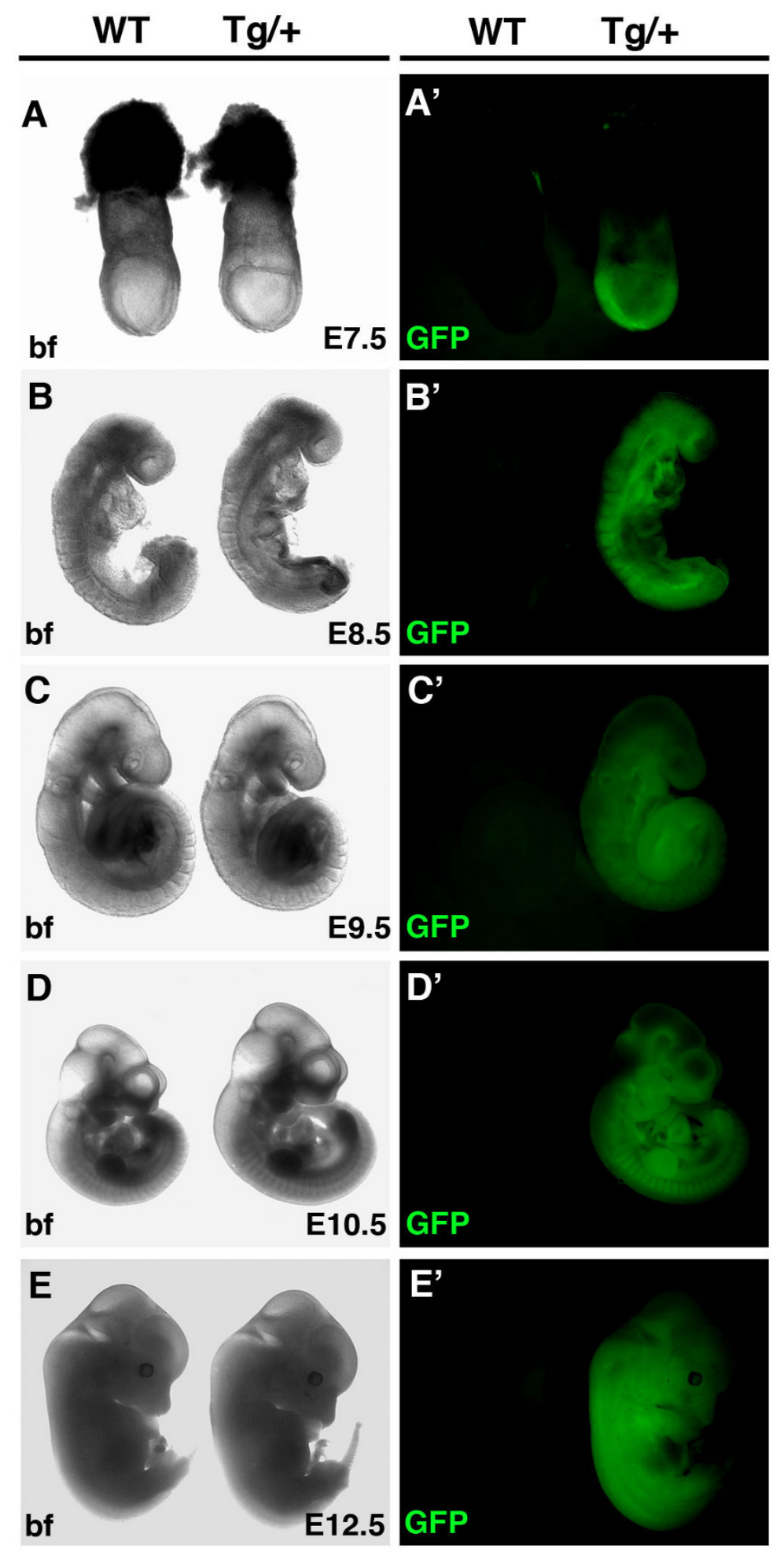

Figure 6

Widespread KikGR expression in CAG::KikGRTg/+ embryos. Panels of bright field and corresponding dark field epifluorescent images of CAG::KikGRTg/+ embryos and non-transgenic littermates at E7.5 (A, A'), E8.5 (B, B'), E9.5 (C, C'), EI 0.5 (D, D') and $\mathrm{EI} 2.5\left(\mathrm{E}, \mathrm{E}^{\prime}\right)$. 


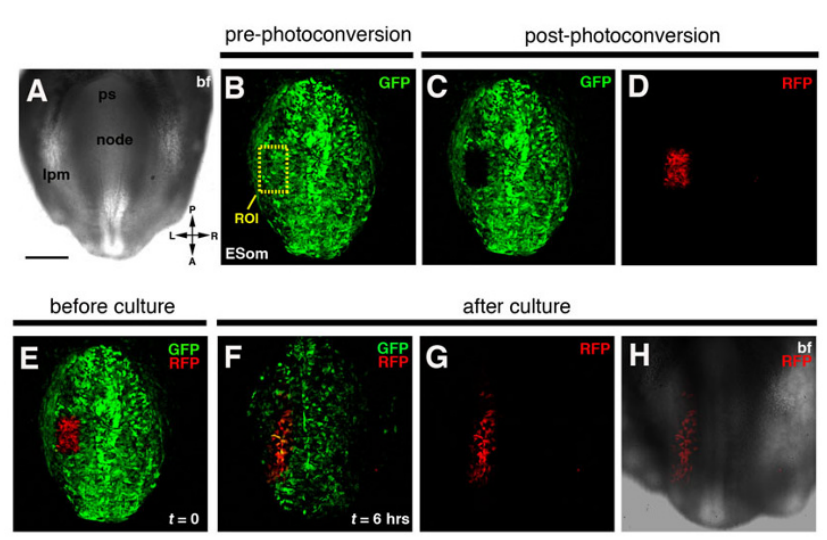

Figure 7

Photoconversion of cells in the lateral plate mesoderm (Ipm) of an E8.0 CAG::KikGRTg/+ embryo: live imaging of behaviors of cell cohorts. Panels show 2D (bright field) and 3D (green and red channels) laser scanning confocal images of a posterior view of an E8.0 CAG::KikGRTg/+ embryo (A-H). Bright field image (A). KikGR expression in an E8.0 embryo prior to photoconversion. Yellow dashed rectangle, region of interest (ROI), demarcates population of cells to be excited with laser light of $405 \mathrm{~nm}$ wavelength (B). Loss of green fluorescence in boxed $\mathrm{ROI}$ after photoconversion (C). Cells in boxed ROI fluoresce red after photoconversion (D). Merge of red and green channels after photoconversion showing labeled lpm cells at start of embryo culture $(E)$. Panels show migration of photoconverted cells in lpm of embryo after $6 \mathrm{hrs}$. Dimensions of cell population photoconverted have become longer and thinner (F-G). Merge of green and red channel (F). Red channel (G). Overlay of red channel onto bright field image $(\mathrm{H})$. ESom, early somite stage; ps, primitive streak. Scale bar indicates $200 \mu \mathrm{m}$

from the early headfold stage (EHF - E7.75) to the early somite stage (ESom) that is equivalent in timing and morphology to wild type in utero development (Figure 9). The image sequence depicts co-ordinate morphogenetic events including the invagination of the foregut pocket, development of the headfolds and condensation of the rostral somites. The group of photoconverted cells retains red fluorescence and can be followed throughout the 10hour time-lapse and culture period. These data reveal the dynamics and expansion of a population of mesodermal cells migrating into the cardiac crescent (see Additional file 1).

\section{Conclusion}

We have compared four widely available photomodulatable fluorescent proteins: PA-GFP which photoactivates to produce green fluorescence, the cyan-to-green PS-CFP2, and Kaede and KikGR, two green-to-red photoconvertible fluorescent proteins. Our data demonstrate that the photomodulatable fluorescent protein KikGR is suitable for a number of live imaging applications in ES cells and in mouse embryos. KikGR is developmentally-neutral, does not autoactivate after exposure to broad spectrum white light, undergoes rapid and complete photoconversion when exposed to light of a specific wavelength, and exhibits bright fluorescence before and after photoconversion.

$C A G:: K i k G R$ transgenic mice lend themselves to a variety of live imaging applications for investigating individual cell behaviors, population dynamics and cell fate in the mouse embryo. Defined ROIs permit high temporal and spatial specificity for cell labeling, yielding high-resolution information on cell dynamics in vivo. Having established widespread expressing KikGR strains and demonstrated their utility, the development of strains exhibiting lineage-specific KikGR should in the future should provide greater spatial specificity for cell labeling. Moreover, the future development of mouse strains expressing subcellularly-localized KikGR or monomeric KikGR fusion proteins should provide greater spatial resolution for live imaging.

\section{Methods}

\section{Plasmids and constructs}

CAG::KikGR was generated by PCR of KikGR from pKIkGR1-S1 (MBL), (GenBank Accession number: AB193293) [25] using oligos EcoRI-KikGR_5': GTTACCGGA ATTCCGGATGGTGAGTGTGATTACATCAGAA and EcoRI-KikGR_3': CAATCCGGAATTCCGGTTACTTGGC CAGCCTTGGCAGCCCGGA. The resulting PCR product was cut with EcoRI cloned into pCAGGS [26]. CAG::PAGFP was generated by PCR of PA-GFP-N1 [27] using oligos EcoRI-PAGFP_5': GTTACCGGAATTCCGGATGGTGAGCAAGGGCGAGGAGCTG and EcoRI-PAGFP_3': CAATCCGGAATTCCGGTTATCTAGATCCGGTGGATC-

CCGG. CAG::PSCFP2 was generated by PCR of PS-CFP2 from $p P S-C F P 2-N$ (Evrogen) [32] using oligos EcoRIPSCFP2_5': GTTACCGGAATTCCGGATGAGCAAGGGCG CCGAGCTGTTC and ECORI-PSCFP2_3': CAATCCGGAATTCCGGTTACTTGTACAGCTCATCCATGCC. The resulting PCR products for each vector were cut with EcoRI and cloned into pCAGGS [26]. CAG::Kaede was generated by PCR of Kaede from pCS2-Kaede [29] using oligos Kaede5F-EcoR1: CCGGAATTCCGG ATGGTGAGTCTGATTAAA CCAGAAATGAAG and Kaede-3R_EcoR1: CCGGAATTCCG GTTACTTGACGTTGTCCGGCAATCCAGAATG. The resulting PCR products for each vector were cut with EcoRI and cloned into pCAGGS [26].

\section{Generation of transgenic ES cells}

All constructs were tested for fluorescence in COS7 cells and R1 ES cells [33]. R1 ES cells were maintained under standard conditions [31]. Transgenic ES cell lines constitutively expressing $C A G:: K i k G R$ were generated by co-electroporation of SalI linearized CAG::KikGR construct and a 

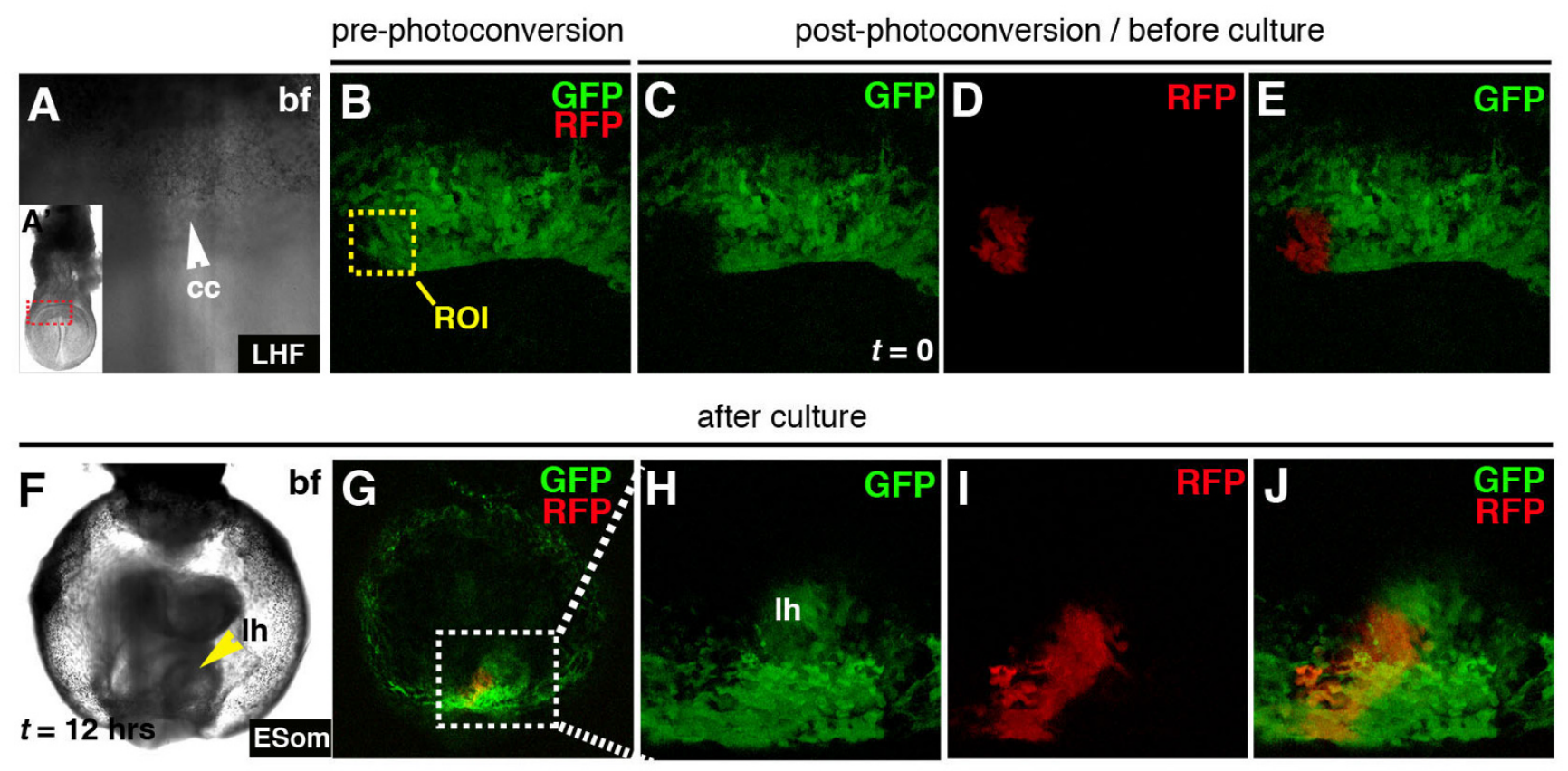

\section{Figure 8}

Live imaging cell fate during mouse cardiac morphogenesis. Panels show 2D (bright field) and 3D (green and red channels) laser scanning confocal images of photoconversion cells on the right side (ROI) of the cardiac crescent (cc) of an E7.5 CAG::KikGRTg/+ embryo (A-E). After 12 hrs of roller culture the embryo has formed a linear heart tube (Ih) and photoconverted cells can be detected on the right side of the linear heart tube (F-J). Widefield image of an E7.5 embryo. Red dashed box shows region of cc depicted in panels $A-E\left(A^{\prime}\right)$. Bright field image of cardiac crescent before culture $(A)$ and embryo after culture $(F)$. Red and green channel (B, E, G and J). Green channel ( $C$ and $H)$. Red channel (D and I). White dashed box in panel $G$ shows region depicted in panels $\mathrm{H}-\mathrm{J}$.

circular PGK-Puro-pA plasmid conferring transient puromycin resistance. Puromycin selection was carried out exactly as described previously [8].

\section{Mouse breeding}

CAG::KikGR was linearized with Sall and gel-purified using routine protocols [31] DNA was injected into C57BL/6 zygotes at the Memorial Sloan-Kettering Cancer Center Transgenic Core Facility. Fo founders were screened by PCR and expression of green fluorescence, resulting in 3 founder lines. Founders were mated to ICR females to recover embryos and subsequent F1 generation adults.

\section{Embryo collection and culture}

Post-implantation embryos and organs were dissected in DMEM/F12 containing 5\% fetal calf serum and cultured in media comprising 50\% rat serum, 50\% DMEM/F12 supplemented with 1\% L-glutamine and 1\% Penicillin/ Streptomycin.

\section{Image acquisition}

All images of mouse embryos or organs presented in the figures are of living embryos or freshly dissected (unfixed) tissues maintained under physiological conditions. ES cells were also imaged live. Wide-field images were acquired on a Leica MZFLIII stereo dissecting microscope or Zeiss Axiovert $200 \mathrm{M}$ inverted microscope equipped with epifluorescent illumination using appropriate filter sets. Laser scanning confocal data was acquired using a Zeiss LSM510 META scan head fitted onto a Zeiss Axiovert $200 \mathrm{M}$. Fluorophores were excited with a $488 \mathrm{~nm}$ Argon laser line (green) and a $543 \mathrm{~nm}$ HeNe laser (red). Objectives used were a plan-apochromat $20 \times / 0.75$ and a planapochromat $10 \times / 0.45$. Confocal images were acquired as sequential optical $x-y$ sections taken at $1-2 \mu \mathrm{m} z$ intervals.

\section{Photoconversion}

COS-7 cell expressing PS-CFP2, KikGR and Kaede were visualized under standard conditions for visualization of CFP and GFP, respectively using a 458 Argon laser (45\%) and a $488 \mathrm{~nm}$ Argon laser (5\% power), respectively, with a plan-apochromat 20×/0.75 objective. A $405 \mathrm{~nm}$ Diode laser (set at 35\% power) was used for photoconversion of COS7 cells in culture with $1 \times 50$ iterations. Photoconverted KikGR and Kaede were imaged using a $543 \mathrm{~nm}$ HeNe laser ( $80 \%$ power) for red fluorescence excitation. Both photoconverted and unconverted KikGR and Kaede 


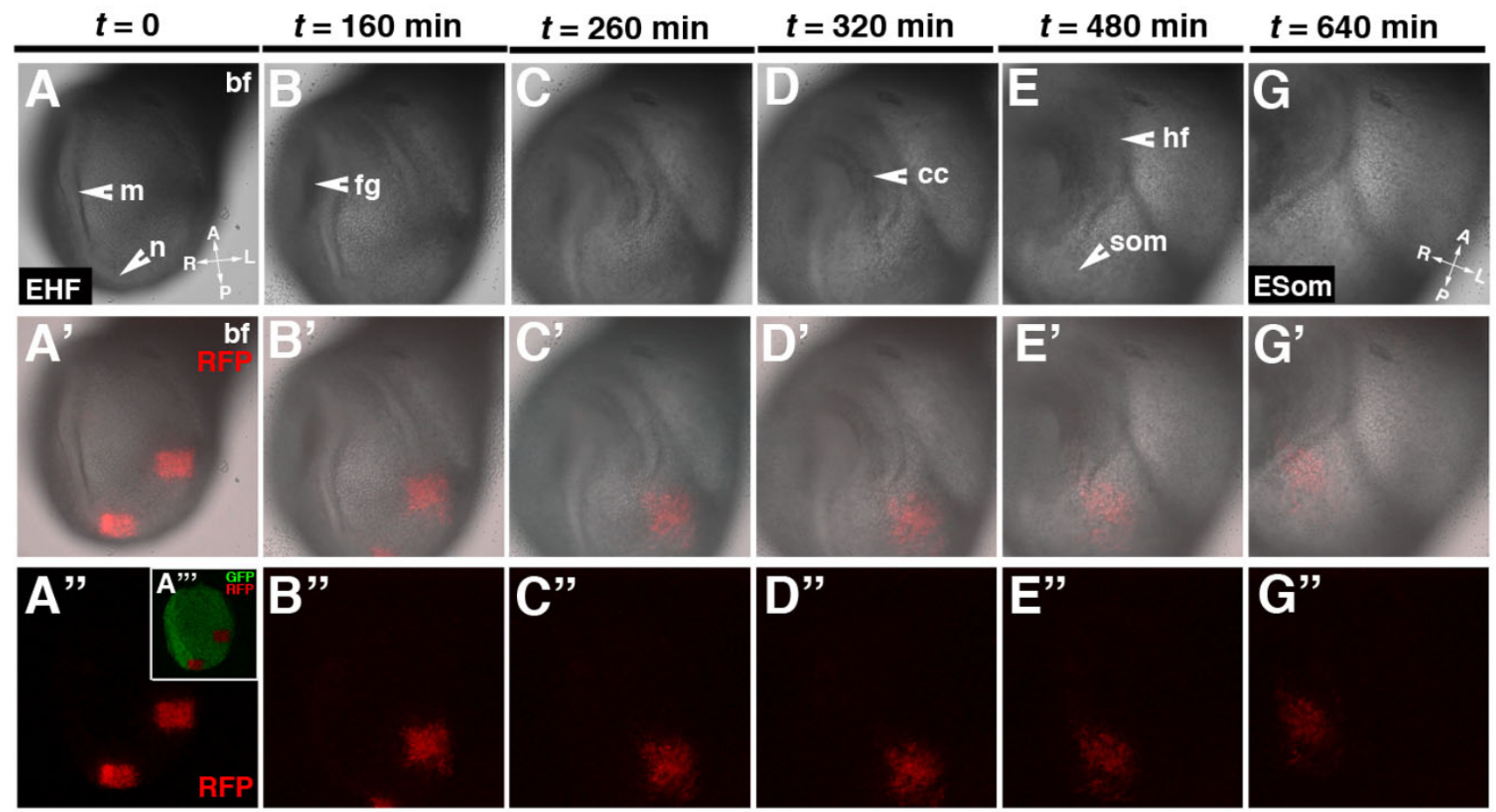

\section{Figure 9}

Live imaging photoconverted KikGR-positive cells in a mouse embryo: photoconversion, ex utero culture and 3D time-lapse laser scanning confocal imaging do not affect embryonic development. Panels show sequence 3D stills over a time of 640 min of a CAG::KikGRTg/+ embryo developing from an early head fold stage to an early somite stage (A-G). The series depicts the developing cardiac crescent (cc), headfolds (hf) and condensing somites (som). Two regions of photoconverted cells are shown over time. Cells of photoconverted region located in the left side of the embryo migrate anteriorly into the cardiac crescent. Bright field images (A-G). Merge of RFP channel and bright field (A'-G'). RFP channel (A"-G"). GFP and RFP channel ( $\left.\mathrm{A}^{\prime \prime}\right)$. $\mathrm{m}$, midline; $\mathrm{n}$, node.

were imaged using $488 \mathrm{~nm}$ Ag laser (5\% power) for green fluorescence and $543 \mathrm{~nm}$ HeNe laser (80\% power) for red fluorescence in multi-track mode. Photoconverted PSCFP2 was imaged using a 458/488 nm Argon laser (45\%/ $5 \%$ laser power) for cyan and green fluorescence respectively, in multi-track mode. Cells and embryos expressing KikGR were visualized under standard conditions for visualization of GFP, using a $488 \mathrm{~nm}$ Argon laser (5\% power) with a plan-apochromat $20 \times / 0.75$ objective. A $405 \mathrm{~nm}$ Diode laser (set at $100 \%$ power) was used for photoconversion of ES cells in culture with $1 \times 50$ iterations, and cells in vivo in mouse embryos with $1 \times 75$ iterations. Photoconverted KikGR was imaged using a 543 $\mathrm{nm}$ HeNe laser (80\% power) for red fluorescence excitation. Both photoconverted and unconverted KikGR were imaged using $488 \mathrm{~nm} \mathrm{Ag} \mathrm{laser} \mathrm{(5 \%} \mathrm{power)} \mathrm{for} \mathrm{green} \mathrm{fluo-}$ rescence and $543 \mathrm{~nm}$ HeNe laser (80\% power) for red fluorescence in multi-track mode. For time-lapse imaging after photoconversion, $488 \mathrm{~nm}$ and $543 \mathrm{~nm}$ lasers $(3 \%$ and $50 \%$ power, respectively) excitation, were used with plan-apochromat $10 \times / 0.45$ or plan-apochromat $20 \times / 0.75$ objectives capturing $512 \times 512$ pixels per frame. For quantifying the photoefficiency of KikGR continuous exposure at $405 \mathrm{~nm}$ (30\% laser power; $25.0 \mathrm{~mW}$ ) was used.

\section{Image processing}

Raw data was processed using Zeiss LSM software (Carl Zeiss Microsystems at http://www.zeiss.com/) and Adobe Photoshop CS2 (Adobe Systems, Inc., San Jose). Re-animation of data to generate movies of time-lapses was performed using QuickTime Pro (Apple Computer, Inc at http://www.apple.com/quicktime/).

\section{Authors' contributions}

$\mathrm{SN}$ - conceived, designed and carried out the experiments, analyzed the results and drafted the manuscript. AKH conceived, funded and supervised the project. Both authors critically read and revised the manuscript, and approved the final version. 


\section{Additional material}

\section{Additional file 1}

Live imaging and photoconversion of KikGR, a green-to-red fluorescent protein, in a mouse embryo. 3D time-lapse of an E7.5 (early headfold stage - EHF) CAG::KikGR embryo from the EHF - ESom (early somite stage). KikGR is a green-to-red photoconvertible fluorescent protein. In the CAG::KikGR strain all cells express KikGR. There are three consecutive sequences in the movie. The first depicts the bright field channel alone to illustrate normal development (foregut invagination, development of the headfolds as well as somite condensation), second the red fluorescent channel (post conversion) overlayed on bright field, and third the red fluorescent channel. Two square regions of interest (ROIs) have been converted within the mesoderm. Each frame depicting fluorescence represents a $3 D$ reconstruction of a $z$-stack $(266 \mu \mathrm{m})$. The interval between slices is $7 \mu \mathrm{m}$. The time interval between $z$-stacks is $20 \mathrm{~min}$. The time-lapse sequence covers 9 hours and $20 \mathrm{~min}$. The embryo is suspended from its ectoplacental cone using a human eyelash. Anterior of the embryo is to the top left.

Click here for file

[http://www.biomedcentral.com/content/supplementary/1471213X-9-49-S1.mov]

\section{Acknowledgements}

We thank the MSKCC Mouse Genetics Core facility for production of transgenic mice; Jennifer Lippincott-Schwartz and George Patterson for the PA-GFP plasmids; Paul Kulesa and Danny Stark for advice on photoactivation; Rodrigo Fernandez-Gonzalez for assistance with MatLab; Ann Foley for comments on the manuscript. Work in our laboratory is supported by the National Institutes of Health (ROI-HD052 II5) and NYSTEM. SN is supported by an American Heart Association postdoctoral fellowship.

\section{References}

I. Haas K, Sin WC, Javaherian A, Li Z, Cline HT: Single-cell electroporation for gene transfer in vivo. Neuron 200I, 29:583-9I.

2. Bronner-Fraser M, Fraser SE: Cell lineage analysis reveals multipotency of some avian neural crest cells. Nature 1988, 335:161-4

3. Kinder S, Tsang TE, Quinlan GA, Hadjantonakis AK, Nagy A, Tam PP The orderly allocation of mesodermal cells to the extraembryonic structures and the anteroposterior axis during gastrulation of the mouse embryo. Development 1999, 126:469I-70 I.

4. Kinder SJ, Tsang TE, Wakamiya M, Sasaki H, Behringer RR, Nagy A, Tam PP: The organizer of the mouse gastrula is composed of a dynamic population of progenitor cells for the axial mesoderm. Development 200।, I 28:3623-34.

5. Eakin GS, Hadjantonakis AK: Production of chimeras by aggregation of embryonic stem cells with diploid or tetraploid mouse embryos. Nat Protoc 2006, I: I I45-53.

6. Le Douarin NM: The ontogeny of the neural crest in avian embryo chimaeras. Nature 1980, 286:663-9.

7. Stern CD, Keynes RJ: Interactions between somite cells: the formation and maintenance of segment boundaries in the chick embryo. Development 1987, 99:26I-72.

8. Hadjantonakis AK, Gertsenstein M, Ikawa M, Okabe M, Nagy A: Generating green fluorescent mice by germline transmission of green fluorescent ES cells. Mech Dev 1998, 76:79-90.

9. Nowotschin S, Eakin GS, Hadjantonakis AK: Live-imaging fluorescent proteins in mouse embryos: multi-dimensional, multispectral perspectives. Trends Biotechnol 2009, 27:266-76.

10. Hadjantonakis AK, Papaioannou VE: Dynamic in vivo imaging and cell tracking using a histone fluorescent protein fusion in mice. BMC Biotechnol 2004, 4:33.

II. Nowotschin S, Eakin GS, Hadjantonakis AK: Dual transgene strategy for live visualization of chromatin and plasma mem- brane dynamics in murine embryonic stem cells and embryonic tissues. Genesis 2009, 47:330-6.

12. Plusa B, Piliszek A, Frankenberg S, Artus J, Hadjantonakis AK: Distinct sequential cell behaviours direct primitive endoderm formation in the mouse blastocyst. Development 2008, 135:308I-9|.

13. Rhee JM, Pirity MK, Lackan CS, Long JZ, Kondoh G, Takeda J, Hadjantonakis AK: In vivo imaging and differential localization of lipid-modified GFP-variant fusions in embryonic stem cells and mice. Genesis 2006, 44:202-I8.

14. Joyner AL, Zervas M: Genetic inducible fate mapping in mouse: establishing genetic lineages and defining genetic neuroanatomy in the nervous system. Dev Dyn 2006, 235:2376-85.

15. Wacker SA, Oswald F, Wiedenmann J, Knochel W: A green to red photoconvertible protein as an analyzing tool for early vertebrate development. Dev Dyn 2007, 236:473-80.

16. Stark DA, Kulesa PM: In vivo marking of single cells in chick embryos using photoactivation of GFP. Curr Protoc Cell Biol 2005, Chapter I 2(Unit I 2):8.

17. Pantazis P, M Gonzalez-Gaitan: Localized multiphoton photoactivation of paGFP in Drosophila wing imaginal discs. J Biomed Opt 2007, I 2:044004

18. Murray MJ, Saint R: Photoactivatable GFP resolves Drosophila mesoderm migration behaviour. Development 2007, 134:3975-83.

19. Caron SJ, Prober D, Choy M, Schier AF: In vivo birthdating by BAPTISM reveals that trigeminal sensory neuron diversity depends on early neurogenesis. Development 2008, 1 35:3259-69.

20. Tomura M, Yoshida N, Tanaka J, Karasawa S, Miwa Y, Miyawaki A, Kanagawa $O$ : Monitoring cellular movement in vivo with photoconvertible fluorescence protein "Kaede" transgenic mice. Proc Natl Acad Sci USA 2008, I 05: I087 I-6.

21. Kurotaki Y, Hatta K, Nakao K, Nabeshima Y, Fujimori T: Blastocyst axis is specified independently of early cell lineage but aligns with the ZP shape. Science 2007, 3 16:719-23.

22. Kulesa PM, Teddy JM, Stark DA, Smith SE, McLennan R: Neural crest invasion is a spatially-ordered progression into the head with higher cell proliferation at the migratory front as revealed by the photoactivatable protein, KikGR. Dev Biol 2008, 316:275-87.

23. Stark DA, Kulesa PM: An in vivo comparison of photoactivatable fluorescent proteins in an avian embryo model. Dev Dyn 2007, 236: $1583-94$

24. Shigematsu Y, Yoshida N, Miwa Y, Mizobuti A, Suzuki Y, Tanimoto Y, Takahashi S, Kunita S, Sugiyama F, Yagami K: Novel embryonic stem cells expressing tdKaede protein photoconvertible from green to red fluorescence. Int J Mol Med 2007, 20:439-44.

25. Tsutsui H, Karasawa S, Shimizu H, Nukina N, Miyawaki A: Semirational engineering of a coral fluorescent protein into an efficient highlighter. EMBO Rep 2005, 6:233-8.

26. Niwa H, Yamamura K, Miyazaki J: Efficient selection for highexpression transfectants with a novel eukaryotic vector. Gene 1991, 108:193-9.

27. J Lippincott-Schwartz, N Altan-Bonnet, Patterson GH: Photobleaching and photoactivation: following protein dynamics in living cells. Nat Cell Biol 2003:S7-I4.

28. Chudakov DM, Lukyanov S, Lukyanov KA: Tracking intracellular protein movements using photoswitchable fluorescent proteins PS-CFP2 and Dendra2. Nat Protoc 2007, 2:2024-32.

29. Hatta K, Tsujii H, Omura T: Cell tracking using a photoconvertible fluorescent protein. Nat Protoc 2006, I:960-7.

30. Galli D, Dominguez JN, Zaffran S, Munk A, Brown NA, Buckingham ME: Atrial myocardium derives from the posterior region of the second heart field, which acquires left-right identity as Pitx2c is expressed. Development 2008, 135: I 157-67.

31. Nagy A, Gertsenstein M, Vintersten K, Behringer R: Manipulating the mouse embryo: a laboratory manual. Cold Spring Harbor Laboratory Press, Cold Spring Harbor, New York; 2003.

32. Chudakov DM, Verkhusha VV, Staroverov DB, Souslova EA, Lukyanov S, Lukyanov KA: Photoswitchable cyan fluorescent protein for protein tracking. Nat Biotechnol 2004, 22: I435-9.

33. Nagy A, Rossant J, Nagy R, W Abramow-Newerly, Roder JC: Derivation of completely cell culture-derived mice from earlypassage embryonic stem cells. Proc Natl Acad Sci USA 1993, 90:8424-8. 